Ann. Abeille, I968, 11 (I), 3I-40.

\title{
EFFET PATHOGÈNE COMPARÉ DE 12 SOUCHES DE BACILLUS THURINGIENSIS BERL. SUR L'ABEILLE DOMESTIQUE ET SON COUVAIN
}

\author{
O. HARAGSIM et Jirina VAŇKOVA \\ Institut de recherches apicoles, Dol par Libcice-sur-la-Moldau (Tchécosloraquie) \\ Laboratoire de pathologie de l'Insecte de l'Institut entomologique, \\ Académie tchécoslozaque des Sciences, Prague (Tchécoslovaquie).
}

\section{SOMMAIRE}

Les auteurs ont comparé l'effet pathogène de 12 souches de Bacillus thuringiensis sur les abeilles, et leur couvain. Les variétés anduze et alesti se sont avérées les plus toxiques pour les butineuses tandis que la souche galleriae était la moins toxique. Dans les expériences sur le couvain d'abeilles la souche bombycis s'est montrée la plus toxique tandis que la souche galleriae ne présentait aucune. toxicité. Simultanément a été étudiée une préparation commerciale tchèque appelée "Bathurin " concentré de spores et d'inclusions de Bacillus thuringiensis var. thuringiensis. A une forte concentration $\left(3 \times 10^{8}\right.$ spores par gramme de nourriture) cette préparation est dangereuse pour les abeilles mais à la concentration de $10^{8}$ on peut la considérer comme inoffensive.

\section{INTRODUCTION}

Bacillus thuringiensis BERLINER constitue un élément prometteur dans la lutte: biologique contre les chenilles des Lepidoptères qui s'attaquent aux produits agricoles, alimentaires ou industriels.

Du point de vue apicole on fait ressortir que Bacillus thuringiensis est un " insecticide vivant " qui n'est pas nuisible pour les abeilles et que son emploi dans 1'agriculture réduit le danger d'intoxication des abeilles par les insecticides chimiques. En particulier, on prévoit son utilisation dans la lutte contre les chenilles de la fausse teigne (Galleria mellonella L.) et de la petite teigne (Achroia grisella $F$.) qui appartiennent, comme on le sait aux parasites les plus dangereux des rayons d'abeilles.

Ce sont les inclusions parasporales protéiniques naissant à l'intérieur des cellules bactériennes à l'époque de la sportulation, qui sont considérées comme vecteurs de 
l'efficacité des souches du groupe de Bacillus thuringiensis contre l'insecte (ANGus I954, I956, I 964, VAŇKová I957). Certaines variétés de Bacillus thuringiensis diffusent dans le milieu de culture, outre l'endotoxine ci-dessus mentionnée, une exotoxine soluble thermostable (McConner, et Richards I959). A l'effet pathogène peuvent également participer la lécithinase et un complexe de protéases.

Au cours des travaux antérieurs (VǍ̌Ková 1965, 1964, I966) nous avons mis en évidence que les souches du groupe de Bacillus thuringiensis exercent une action différente sur les divers Lépidoptères. La virulence différente des souches particulières s'est manifestée de la façon la plus marquée sur la teigne de la cire, Galleria mellonella L. Sur I2 souches examinées, c'est la souche de Bacillus thuringiensis, variété galleriae qui accusait la toxicité la plus élevée pour Galleria mellonella. Pour qu'on puisse utiliser la souche en question et le cas échéant, les souches ultérieures dans la pratique de la lutte biologique, il était nécessaire de mettre en évidence, soit l'innocuité, soit la toxicité des souches particulières à l'égard des abeilles. Les travaux présentés jusqu'ici par les auteurs qui ont étuđié l'influence des différentes souches sur les abeilles concernaient en premier lieu la souche classique de Bacillus thuringiensis, variété thuringiensis Berliner et les souches employées dans l'industrie à la production des préparations Thuricide, Biospor, Biotrol, Entobakterin, Dendrobacillin et Parasporin (Cantweli, et coll., I964, I966, Celli et GiorDani I966, Krieg ig64, Stute I963, Leskova et Kulikov i963, Wilson ig62, Johansen 1962, Poltev 1963). Les effets des différentes souches ne peuvent être comparés que très difficilement, les auteurs utilisant des méthodes d'application différentes. La plupart d'entre eux sont cependant d'accord pour admettre que les préparations à base de Bacillus thuringiensis et des souches parentes ne sont toxiques pour l'abeille qu'à des doses élevées qui ne sont pas utilisées au cours du traitement des plantes sur le terrain.

Le but de notre travail a consisté à déterminer et à comparer l'effet pathogène des I2 souches de Bacillus thuringiensis sur les imagos et le couvain de l'abeille domestique. Les préparations étaient produites et examinées d'une façon uniforme. De toutes ces souches six n'avaient jamais été essayées sur l'Abeille.

\section{MATÉRIEL, ET MÉTHODE}

Les sonches de Bacillus thuringiensis ont été cultivées en milieu liquide aéré et agité, dans des fermentateurs de laboratoire de 20 litres, selon la méthode élaborée pour le Bacillus thuringiensis, variété thuringiensis (VAŇKoVÁ 1958, VAN̆KOVÁ et WEISER 1962). Le matériel sporulé (spores, inclusions et résidus des cellules végétatives) a été séparé du milieu de culture par centrifugation et séché par lyophilisation. Le matériel sec a été dilué au moyen de talc pour ajuster la concentration des spores à $10^{11}$ par gramme.

\section{Origine des souches testées (tabl. I)}

Outre les souches mentionnées on a testé l'effet de la préparation tchécoslovaque dénommée "Bathurin ", ce qui est un concentrat de spores et d'inclusions de Bacillus thuringiensis, variété thuringiensis sans addition de charge.

Au cours des essais les abeilles ont été placées dans un étuve à une température de $30^{\circ} \mathrm{C}$ et une humidité de 60-70 p. 100 et maintenues dans des cagettes de bois de $5 \times 7 \times 15 \mathrm{~cm}$, à raison de 50 dans chaque cagette. L'une des parois de la cagette était formée d'un grillage métallique, l'autre étant en verre. Le nourrisseur de la cagette était en verre; on pouvait introduire dans la cagette la nourriture de l'extérieur, sans pour cela troubler les abeilles. 
FFFE'T PATHOGÈNE DE “ BACILLUS THURINGIENSIS 》

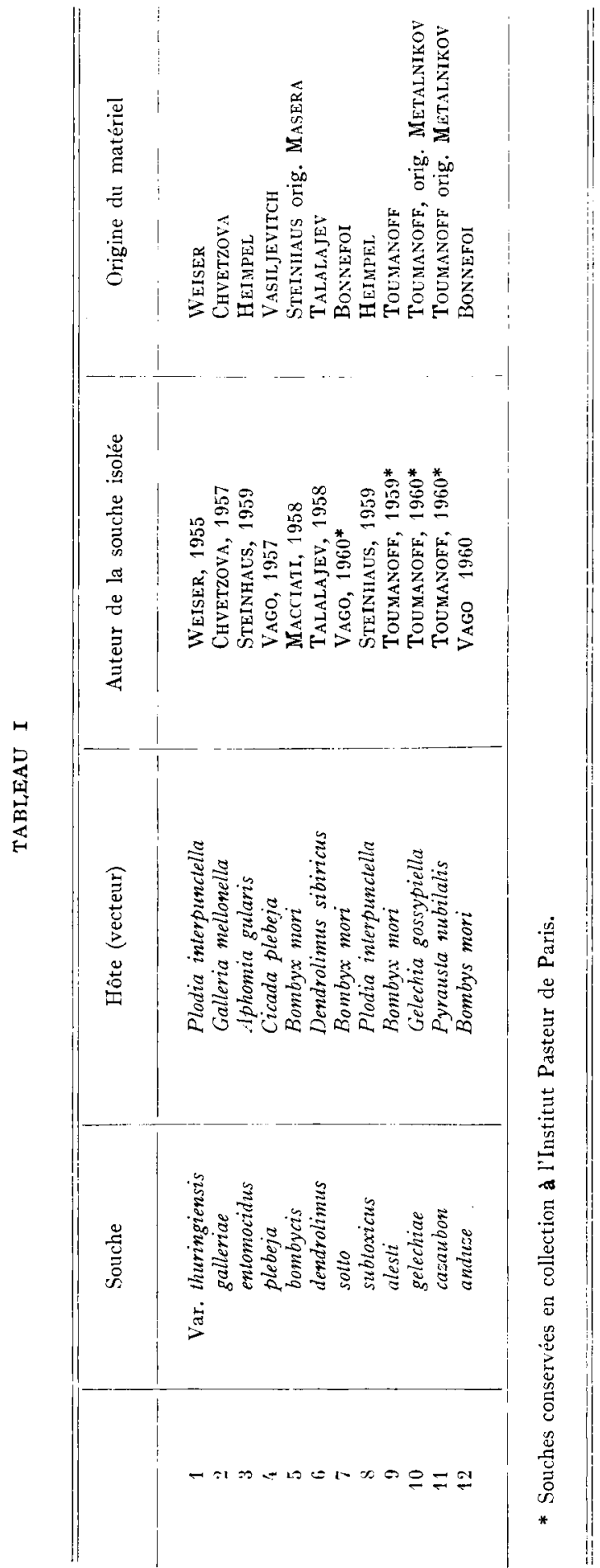

Annales de l'Abeille. - I 968. 
Les abeilles provenaient des colonies de l'Institut de recherches apicoles de Dol et d'après les caractères morphologiques nous les considérons comme les produits du croisement naturel de l'abeille locale (Apis mellifera mellifera $\mathrm{L}$.) avec l'abeille carniolienne (Apis mellifera carnica). On a testé toujours deux séries de butineuses d'âge indéterminé et de jeunes abeilles provenant de la même colonie mais âgées de 6 à 8 jours.

Avant le délbut des essais on a laissé les abeilles a jeun pendant 24 heures. Au cours des essais on les nourrissait de sirop de sucre dans lequel on avait introduit les spores et les inclusions de la souche testée correspondante de Bacillus tharingiensis. Pour choisir la concentration des spores dans la nourriture, on s'est appuyé sur les domées de KRIEG et de IIsRlis (ig6-4) qui indiquent la concentration de spores de $10^{8}$ par gramme de nourriture comme seuil de la dose léthale de Bacillus thuringiensis pour les abeilles. Pendant toute la durée de l'essai (I6 jours) on a nourri les abeilles ad libitum avec le sirop de sucre (1/1) additionné de spores. 1)u pollen cl'arbres fruitiers, conservé dans un petit rayon constituait la composante azotée de la nourriture. Le contrôle des cagettes se faisait tous les jours; on éliminait les abeilles mortes et on distribuait la nourriture fraîche.

Pour vérifier l'effet de Bacillus thuringiensis BERL. sur le couvain d'abeilles, on a pulvérisé du sirop de sucre contenant les spores et les inclusions de la souche correspondante à la concentration de ${ }^{8} 0^{8}$ par gramme, la pulvérisation s'effectuant en une seule fois sur I $\mathbf{d m}^{2}$ de couvain non operculé de deux ou trois jours. L'effet de l'intervention était évalué en comparant les photographies di rayon traité avant et après l'intervention (cellules operculées et abeilles écloses). Lin outre on suivait l'état sanitaire des abeilles en voie d'éclosion et l'époque de l'éclosion.

Les résultats, c'est-à-dire la mortalité, ont été calculés selon la formule d'ABisotr (I925):

$$
\text { M p. 100 }=\frac{(n-k) \cdot \text { Ioo }}{n} \quad \begin{aligned}
& n=\text { mortalité dans l'essai } \\
& k==\text { mortalité chez le témoin }
\end{aligned}
$$

\section{RÉSULTATS}

Il ressort des résultats, résumés dans les tableaux 2 et 3 , que c'est Bacillus thuringiensis, variété anduze isolé par VAGo à partir de Bombys mori qui exerce l'effet toxique le plus important sur les abeilles butineuses. En effet, 43,8 p. Ioo des buti-

TABLEAU 2

Effet toxique des spores et des inclusions de diverses souches de $\mathrm{B}$. thuringiensis sur les imagos des abeilles

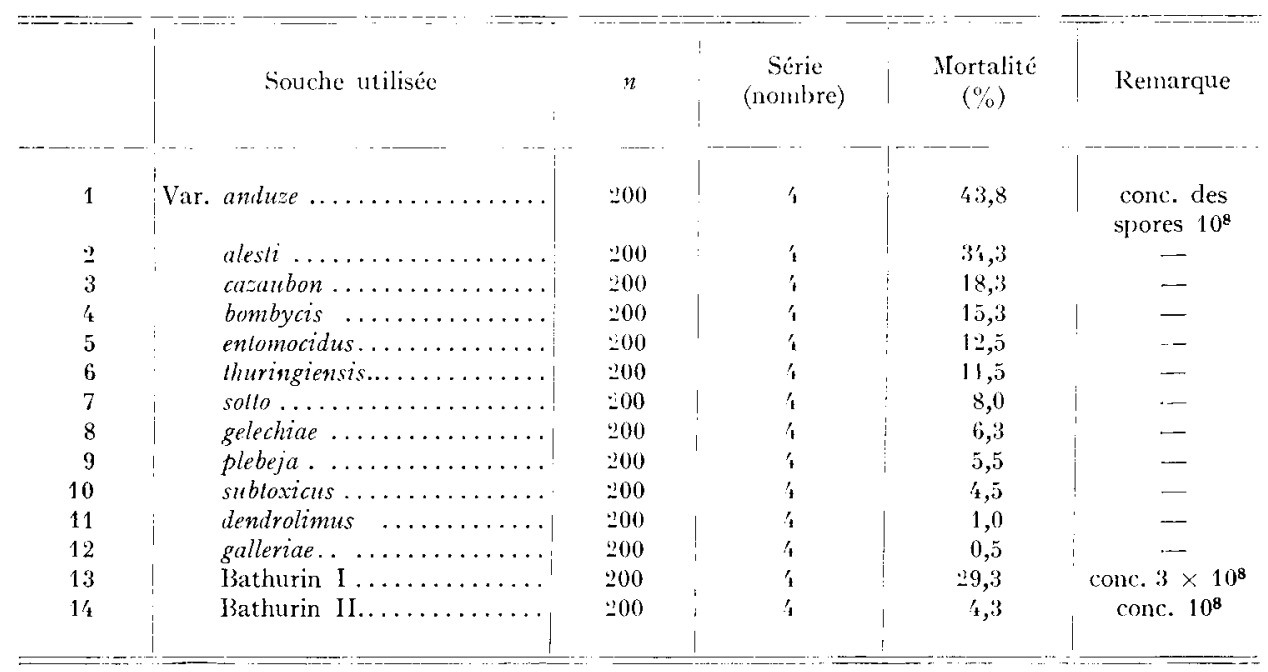


neuses sont mortes au bout des I6 jours de l'essai. La souche alesti se rapproche le plus de la souche anduze, son effet se manifestant sur les abeilles par une mortalité de 34,3 p. I00. Les deux souches appartiennent au groupe dont l'effet pathogène est supérieur à 20 p. Ioo. Un autre groupe est formé par des souches de Bacillus thuringiensis, dont la toxicité s'est manifestée au cours de nos essais par ne mortalité inférieure à 20 p. I00. Ce sont les souches cazaubon (I8,3 p. IOO), bombycis (I 5,3 p. IOO), entomocidus (I2,5 p. IOO), thuringiensis (II,5 p. IOO) qui appartiennent à ce groupe. L'effet toxique de toutes les autres souches ne dépassait pas, quant à la mortalité des abeilles, Io p. Ioo : la souche sotto (8,o p. I0o), gelechiae (6,3 p. roo), plebeja (5,5 p. IOO), subtoxicus (4,5 p. I00), dendrolimus (I,o p. roo), galleriae (0,5 p. I00).

Le "Bathurin " préparation tchécoslovaque du commerce accusait, à une concentration de $3 \times 10^{8}$ un effet toxique causant une mortalité des abeilles égale à $29,3 \mathrm{p}$. Ioo. A une concentration moins élevée, soit $10^{8}$ spores par gramme de nourriture, la mortalité n'a été que de 4,3 p. roo.

\section{TABLEAU 3}

Effet toxique des spores et des inclusions de diverses souches de Bac. thuringiensis sur le couvain d'abeilles

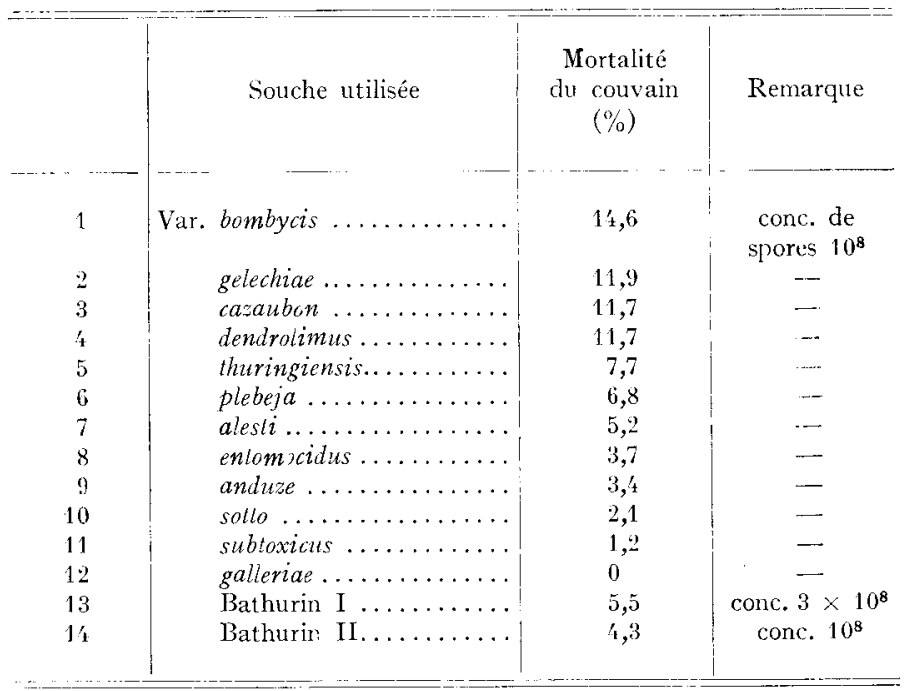

Au cours de nos essais sur le couvain d'abeilles, c'est la souche bombycis, isolée à partir du ver à soie, qui s'est manifestée comme étant la plus toxique. Son action sur le couvain d'abeilles s'est manifestée par une mortalité des larves se chiffrant à I4,6 p. Ioo. L,es souches gelechiae, cazaubon, dendrolimus sont presque identiques en ce qui concerne leur effet toxique sur le couvain d'abeilles (tabl. 3). Leur action s'est manifestée par une mortalité des larves supérieure à Io p. I00. Les autres souches - thuringiensis, plebeja, alesti, entomocidus, anduze, sotto et subtoxicus ont accusé un effet toxique inférieur à Io p. Ioo. Mais la souche galleriae apparaît comme tout à fait dépourvue de toxicité pour les larves des abeilles; elle est également la moins toxique pour les butineuses. 


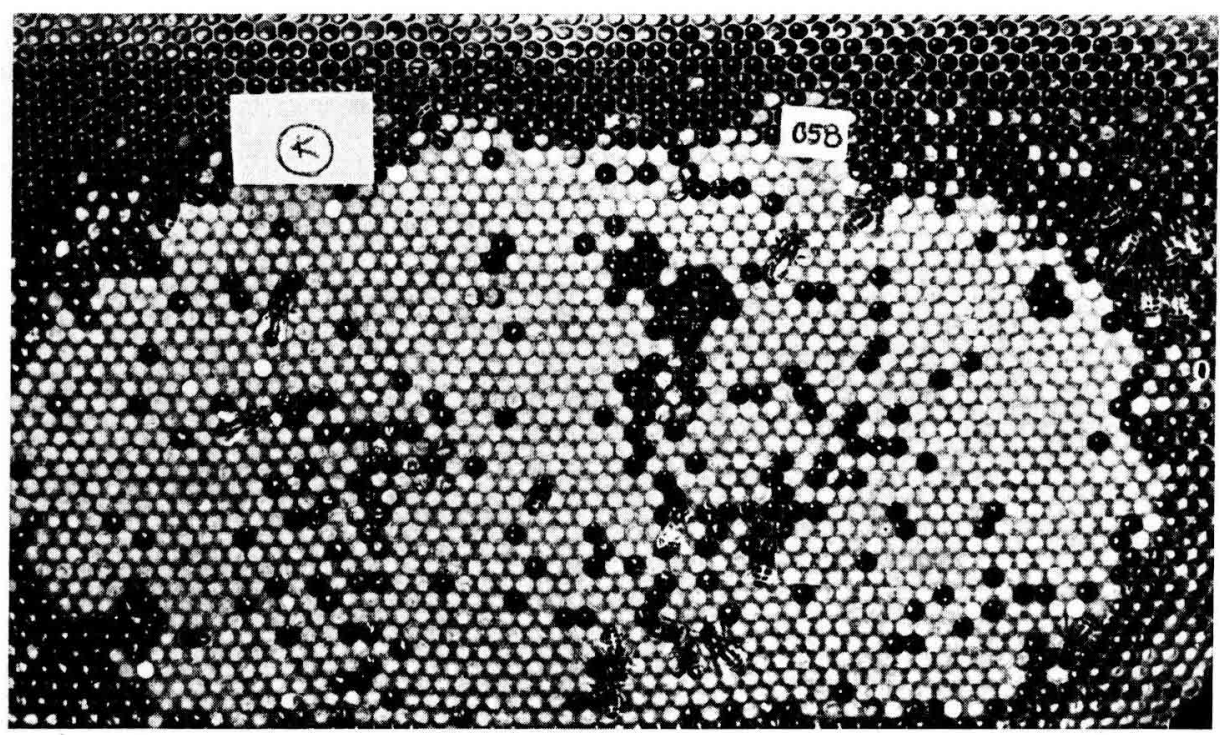

Iir. I. - Rayon avec couvain d'abeilles operculé appès l'application de Bac. thuringiensis, 058 $K=$ Témoin traité seulement par le sirop le sucre sans spores

(photo O. IIaragsim).

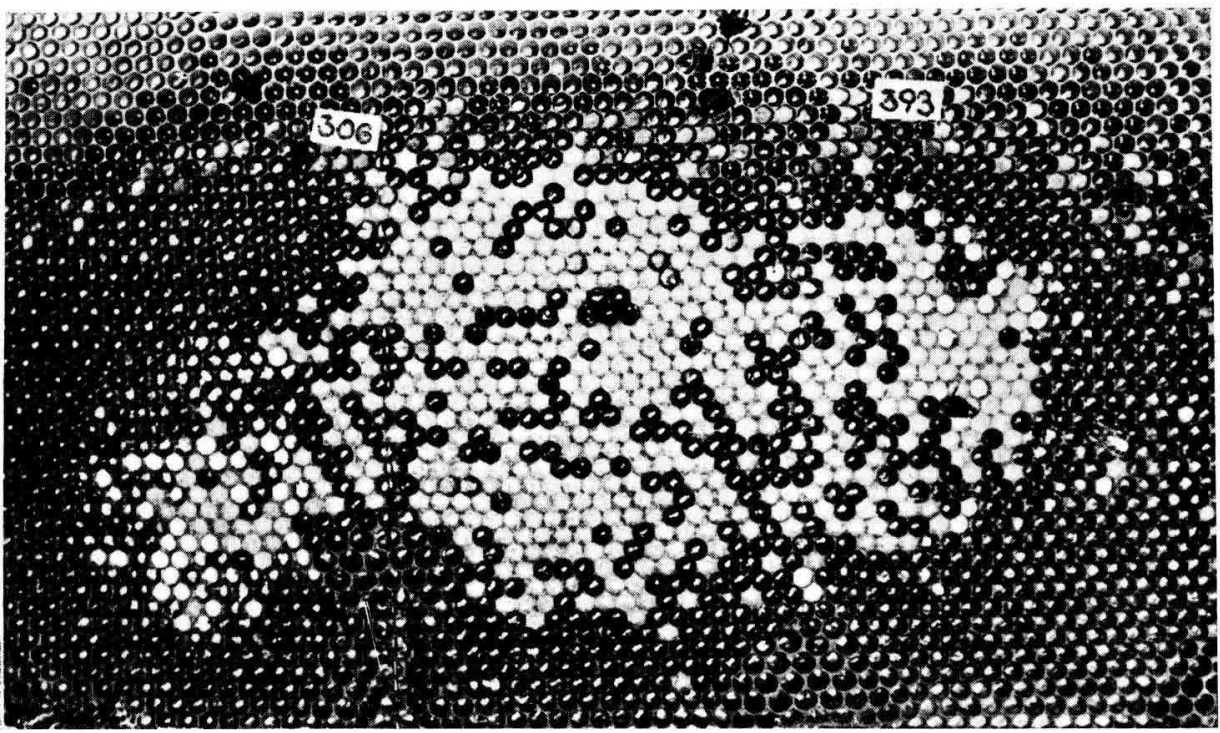

FIr. 2. - Cowvain d'abeilles mourant, traité anant l'operculation

far suspension de spores de bacillus thuringiensis

(conc. $o^{8}$ spores/g dans du sirop de sucre)

Souches 306 : dendrolimus ; 393 : bombycis

(Photo O. IIarausim). 
La préparation "Bathurin " exerçait à la concentration de $3 \times \mathrm{IO}^{8}$ et $\mathrm{IO}^{8}$ sur le couvain d'abeilles un effet toxique faible, la mortalité du couvain étant respectivement de 5,5 p. Ioo et de 4,3 p. Ioo.

Il ressort de nos essais effectués au laboratoire que pour l'abeille domestique ce sont les souches anduze et alesti du groupe de Bacillus thuringiensis qui sont les plus toxiques pour l'abeille domestique. Les autres souches exercent un effet toxique faible, de sorte qu'on peut pratiquement les considérer comme non pathogènes pour les abeilles. L'effet toxique du "Bathurin " ne s'est manifesté sur les abeilles qu'à une concentration élevée de spores et d'inclusions. Cette préparation, à une concentration moins élevée recommandée dans la lutte biologique, apparaît comme une substance non nocive pour les abeilles.

Les abeilles mortes par suite de l'effet toxique de Bacillus thuringiensis ne manifestaient extérieurement aucun symptôme caractéristique d'une infection. Le caractère le plus marqué consistait dans un noircissement de l'exosquelette et une raréfaction des poils sur la face dorsale du thorax. L'exosquelette n'était cependant que d'un noir mat, ce qui le différencie considérablement du noircissement brillant de la chitine, caractéristique du mal noir (" petites noires ») des abeilles.

\section{DISCUSSION}

Les résultats obtenus montrent qu'on peut attribuer aux différentes souches de Bacillus thuringiensis un degré variable de toxicité à l'égard de l'abeille domestique et de son couvain. Le fait que la souche de Bacillus thuringiensis, variété galleriae, qui est la plus virulente contre Galleria mellonella L. (VAŇKoví, Ig66), est par ailleurs la moins toxique pour les butineuses dans la concentration utilisée $(0,5 \mathrm{p}$. Ioo) et parfaitement non toxique pour le couvain d'abeilles, mérite de retenir notre attention.

Poltev (I963) n'a pas pu mettre en évidence une mortalité chez les abeilles nourries avec un sirop de sucre contenant de l'Entobakterin (préparation à base de Bacillus thuringiensis, variété galleriae) pendant un temps de 24 heures à la concentration de I p. Ioo. Au contraire, Lisskova et KULIKov (I963) en procédant au nourrissement ininterrompu des abeilles avec un sirop contenant I gramme de spores par centimètre cube, pendant neuf jours, ont pu mettre en évidence un effet toxique marqué. Fn employant 1'Entobakterin au cours d'essais en plein champ, à la dose de O,I-I,O g par $\mathrm{cm}^{2}$, aucune mortalité des abeilles n'a été constatée. De même en utilisant 10 à $5^{\circ}$ grammes de préparation par $\mathrm{I}^{2}$ de rayons on $\mathrm{n}^{\prime} \mathrm{a}$ pu observer aucun dommage pour les abeilles ou leur couvain. Les travaux en question laissent entrevoir que, même en utilisant une souche identique on ne peut que très difficilement comparer les résultats obtenus par l'application de différentes méthodes. De même les tests de laboratoire ne sont pas tout à fait analogues à ceux de plein champ. Tant que la standardisation internationale des préparations biologiques ne sera pas introduite et cela non seulement en ce qui concerne la fixation du nombre de spores, mais aussi quant aux biotests réalisés sur plusieurs hôtes standards, on doit admettre que les charges des différentes préparations fabriquées par un même producteur, peuvent introduire des différences considérables quant à leur efficacité (CANTWELL et coll., Ig66). 
C'est l'exotoxine non spécifique, qui n'est produite que par certaines souches de Bacillus thuringiensis et qui exerce son action toxique même sur des hôtes sur lesquels les inclusions cristallines restent sans effet, qui pourrait devenir un facteur important dans la pathogénéité des préparations vis-à-vis de l'abeille domestique. Cela dépend du procédé de production utilisé pour la préparation et de la souche employée ainsi que du volume final d'endotoxine que contient la préparation sèche. KRIEG et Herfs ( I 963) ont constaté des mortalités d'abeilles de 60 p. Ioo et de roo p. roo à la suite d'un nourrissement pendant neuf jours à l'exotoxine des souches de Bacillus thuringiensis, variété dendrolimus et de Bacillus thuringiensis, variété thuringiensis, tandis qu'à la suite de la distribution du filtrat des souches euxoae et sotto aucune mortalité n'a eu lieu. MARTOURET et EUVERTE (I964) ont montré que la présence de toxine thermostable dans une préparation commerciale de $B$. thuringiensis var. thuringiensis accroît considérablement la toxicité pour 1'Abeille.

Cantweli, et al. (I966) ont essayé à leur tour l'exotoxine contenue dans une préparation du commerce de Bacillus thuringiensis et ont constaté au bout de quatre jours à des doses plus élevées (20 $\mathrm{mg}$ par $\mathrm{ml}$ de nourriture) une mortalité des abeilles de Ioo $\mathrm{p}$. IO0. A des doses moins élevées $(0,625 \mathrm{mg}$ par $\mathrm{ml}$ de nourriture) pendant cinq jours aucune mortalité n'avait lieu. Sur les larves d'abeilles les doses élevées d'exotoxine exerçaient un effet toxique.

Les préparations utilisées au cours de nos essais, étaient séparées du milieu de culture par centrifugation pendant la production, ce qui a eu pour conséquence l'élimination de la plupart des exotoxines. Les préparations sèches ne pouvaient contenir que de faibles résidus d'exotoxine incapables d'influencer la pathogénéité de la préparation pour l'abeille. La souche de Bacillus thuringiensis, variété thuringiensis, isolée en Tchécoslovaquie et utilisée chez nous à la production de la préparation "Bathurin" ne produit pas d'exotoxine. Fn ce qui concerne la souche de Bacillus thuringiensis, variété galleriae, nous n'avons mis en évidence dans nos conditions de culture qu'une production insignifiante d'exotoxine (VAŇková, I966).

Les différences de toxicité des préparations que nous trouvons dans la bibliographie s'expliquent pour les raisons exposées ci-dessus. Ce qui importe à la plupart des auteurs c'est de déterminer la concentration seuil de toxicité des préparations pour l'abeille domestique. KRIEG et HERFs (I963) indiquent que les doses supérieures à $3 \times 10^{6}$ spores par jour et par abeille occasionnent une mortalité de Ioo p. Ioo. Dans un travail ultérieur (I964) ils augmentent cette dose pour les préparations du commerce "Biospor " Biotrol " et "Thuricide " jusqu'à une concentration de Io à IO ${ }^{8}$ spores par ml de nourriture. GUKASJ AN (I958) a constaté une mortalité de 5o p. roo des abeilles à la suite de l'application d'une préparation de Bacillus thuringiensis, variété dendrolimus à une concentration de $2 \times 10^{9} / \mathrm{ml}$. LECOMTE et MARTOURET (r959) ont déterminé chez la souche anduze une mortalité de Ioo p. Ioo pour une concentration de 80 ooo UB par gramme de candi. Au cours de nos essais ultérieurs cette souche occasionnait une mortalité de $43,8 \mathrm{p}$. Ioo d'abeilles, à la concentration de $\mathrm{I}^{8} / \mathrm{ml}$. Il est intéressant de constater que les deux souches qui étaient au cours de nos essais les plus toxiques pour l'abeille domestique ont été isolées, de Bombyx mori.

On retrouve la même origine (ver à soie) pour la souche bombycis qui s'est manifestée comme étant la plus toxique pour le couvain d'abeilles (mortalité de 14,6 p. I0o) Au contraire, Bacillus thuringiensis, souche galleriae, isolé à l'origine chez Galleria 
mellonella habitant permanent des ruches d'abeilles, est le moins toxique pour l'abeille domestique, qu'il s'agisse des imagos ou du couvain. L'idée qui s'impose, c'est que l'abeille domestique, $\mathrm{du}$ fait qu'elle entre souvent en contact avec Bacillus thuringiensis, variété galleriae, a gagné peut-être une certaine immunité à l'égard du microbe en question.

Reçu pour publication en mai 1968.

\section{SUMMARY}

COMPARATIVE PATHOGENIE EFFECT OH I 2 STRAINS OF " BACILLUS TIIURINGIENSIS " BERL.

ON 'LHE HONEY BEE ANH) ON ITS BROOJ)

I. I) strains of Bacillus thuringiensis BERL. on bees and on their brood. The spore material from the different strains was obtained by fermentation in a liquid medium which was aired and shaken; the spores and inclusions were separated by centrifugation, dried up by lyophilisation and standardized with talc so as to obtain a uniform concentration of spores equal to $10^{11}$ per gramm. For some strains of Bacillus thuringiensis this concentration had been indicated as the threshold of lethal doses.

2. By comparing the pathogenic eflect of the different strains of Bacillus thuringiensis, we found out that the toxicity of the strains for adult bees had the following effects : anduze $(43,8 \mathrm{p}$. 100), alesti (34,3 p. 100), casaubon (18,3 p. 100), bombycis ( 1 5,3 p. 100), entomocidus (12,5 p. 100), thuringiensis (1 1,5 p. 100), sotto (8.0 P. 100), gelechiae $(6,3 \mathrm{P} .100)$, plebeja $(5,5 \mathrm{p} .100)$, subtoxicus $(4,5 \mathrm{P}, 100)$, dentrolimus $(\mathrm{I}, 0$ p. 100), galleriae $(0,5$ P. 100$)$.

3. The strains of Bacillus thuringiensis which have been studied affected the brood as follows : bombycis (14,6 p. 100), gelechiae (I ,9 p. 100), casaubon (1 1.7 p. 100), dendrolimus (I ,7 p. 100), thuringiensis $(7,7$ p. 100), plebeja $(6,8$ p. 100), alesti (5,2 p. 100), entomocidus (3,7 p. 100), anduze $(3,4 \mathrm{p} . \mathrm{I} 00)$, sotto (2, I p. I00), stibtovicus ( $1,2 \mathrm{p} .100)$, galleriae (o p. 100).

4. At the same time, we carricd out investigations on the Czechoslovak commercial preparation called "Bathurin ". This preparation is a concentrate - produced industrially - of spores and inclusions of Bacillus thuringiensis var. thuringiensis, isolated by WEISSER from Plodia interpunctella in 1955. The active part we analyzed contained $3 \times 10^{8}$ spores per gramm of food. At this concentration, "Bathurin " is clangerous for the bees. D)uring our trials, we noted a death-rate of 29,3 P. I OO. At a concentration of Io "Bathurin " only has a low toxic effect on the bees and on their brood (the death-rate amounts 104,3 p. 100), so that it can be considered, at that concentration, as non-rlangrerous for the bees.

\section{RÉFÉRENCES BIBLIOGRAPHIQUES}

Axis:s T. A., 1954. A bacterial toxin paralyzing silkworm larvae. Valure, 173, 545 .

ANGLs T. A., I956. Extraction, purification and properties of Bacillus sotto toxin. Canad. J. Microbiol, $2,416-426$.

Anges 'T. A., I964. The biochenistry and mode of action of Bactllus thuringiensis Berl, and its varieties. Entomophaga, R.-s., no $2,165-17.3$.

Barjac II. de, Bonnefor A., Io62. Essai de classitication biochimique et sérologique de 24 souches de Bacillus du type $B$. thuringiensis. Entomophaga, 7, 5-31.

BaRJAC II. de, Bonneror A., 1967. Classification des souches de Bacillus thuringiensis. Entomophaga, C. R. Lad. Sci. Paris, 264, $181 \mathrm{I}-1 \mathrm{~s} 13$.

Cantwell (G. E., KNox D. A., Michael A. S., ig64. Mortality of honeybees (Apis mellifera L.), fed with exotoxin of Bac. thuringiensis BERL. var. Ihuringiensis. J. Insect. Pathol., 6, $53^{2-536 .}$ 
Cantwell G. E., Knox D. A., Lehnert T., Michael, A. S., ig66. Mortality of the honey bee (Apis mellifera L.) in colonies treated with certain biological insecticides. J. Invert. Pathol, 8, 228-233.

Celli G., GIORDANI G., s 966 . Ricerche sull'attivita del Bacillus thuringiensis Berliner in riguardo all' Apis mellijera J.. Boll. Ist. Enlomol. Univ. Bologna, 28, 141-175.

Gukasjan A. 33., 1958. La sensibilité des abeilles vers le provocateur de la maladie de Denlrolimus sibiricus (orig. russe). Pchelovodstvo, $\mathbf{3 5}$ ( I 1), 46-48.

Johanson C., 1962. Impregnated foundation for Wax moth control. Glean. Bee Cult., 90, 682-684.

KRIEG A., 1964. ¿ iber die Bienenverträglichkeit Industrie-Präparate des Bacillus thuringiensis. Ans. Schädlingskde, 37, 39-40, 1964 .

KRIEG A., HERFS W., 1963. Empfindlichkeit verschiedener Insektenarten gegenüber dem "Exotoxin" von Bacillus thuringiensis BerL. Z. Pflanzenhrankh. Pfansenschutz, 70, I1-21.

KrIEG A., Herfs W., ig63. Ueber die Wirkungen von Bacillus thuringiensis auf Bienen. Entomol. exper. appl., 6, I-9.

KrIEg A., HerFs W., 1967 . Nebenwirkungen von Bacillus thuringiensis Einwirkungen auf Bienen (.Apis mellifera L.). Entomophaga, h.-s. n0 $2,193^{-195}$.

Lecomte J., Martouret D., 1959. Non toxicité pour les ableilles des traitements à base de Bacillus thuringiensis, souche anduze. Ann. Abeille, 2, $17^{-1} 75$.

Leskova A. V., Kul.rkov N. S., 1963. L'influence d'entobacterin et thuricide sur les abeilles (orig. russe). Pchelon'odsivo, 40/3 32-33, 1963.

Martouret D., Euverte G., 1964. The effect of Bacillus thuringiensis BERLINer preparations on the honey bee under conditions of forced feeding. J. Insect Palhol., 6, I $98-203$.

MCCONnel E., RICIIARds A. G., I959. The prcduction by Bacillus thuringiensis Beri. of a heat-stable substance toxic for insects. Canad. J. Microbiol., $5,161-168$.

Poltev V. I., j 963 . Les méthcdes microbiologiques de la lutte contre l'insecte nuisible dans l'apiculture. I ${ }^{\mathrm{e}}$ Congr. internation. Apicult. Prague, 107.

Stute K., נ963. Ueber die Wirkung von Bacillus thuringiensis auf die Ifonigbiene (Apis mellifera I..). Nachrbl. disch. Pflanzenschutedienstes, Braunschreig, 15, 102-104.

VAŇKová J., I957. Study on the effect of Bacillus thuringiensis on insects Folia biol., 3, I 75-182.

VAŇková J., I958. Kultivierung von Bacillus thuringiensis im Versuchsbetriebsmasstab. Trans. Ist. internation. Conf. Insect Palhol., l'rague, 59-64.

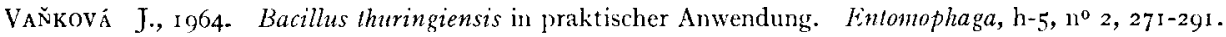

VAN̆Koví J., I 965. Pathogenität einzelner Stänme der Gruppe Bacillus thuringiensis für verschiedene Lepidopteren-Vertriter. Proc. 12 inlernation. Congr. Entomol. London, 1964, 739 .

VAŇ̃koví J., 1966. Pathogenicity of different st rains of Bacillus thuringiensis group for Galleria mellonella $\mathrm{L}$. Acta entomol. Bohemoslovaca, 63, г о-1 6.

VANKovi J., WEISER J., I 962 . I'reparation of insecticidal bacterial preparation from crystal-forming bacilli of the group Bacillus thuringiensis. Czechoslovak patent 105. 4:6, P'rague I 962.

WILSON W. F., I962. Observation on the effects of feeding large quantities of Bacillus thuringiensis BERL. to honey bee. I. Insect. Pathol., 4, 269-270. 